\title{
MODELO DE GESTIÓN PARA HOMOGENEIZAR Y ASEGURAR CALIDAD DOCENTE EN UNA UNIVERSIDAD CON EXPANSIÓN TERRITORIAL
}

\section{Introducción}

La Universidad Arturo Prat, creada en 1984, heredó de la Universidad de Chile un estado de desarrollo incipiente, caracterizado por una función orientada principalmente a la docencia, con escasa oferta académica, número reducido de alumnos, asignación en aporte fiscal directo limitado $(0,47 \%$ del total), marcado déficit en infraestructura y equipamiento, cuerpo académico con insuficiente nivel de perfeccionamiento superior e incipiente actividad de investigación y extensión.

No obstante lo anterior, es una organización que -por la relación que se generó históricamente entre el anhelo de consolidarse como una universidad compleja (es decir, que desarrolla docencia de pre y posgrado, investigación y extensión) con capacidad competitiva y su debilitada situación financieraoptó por un modelo de gestión emprendedor, que consideró entre sus variables constructivas tener una capacidad creativa permanente, alta capacidad de trabajo, buena afinidad para descubrir las necesidades de educación superior del mercado y alta flexibilidad en su gestión.

Todo lo anterior la llevó a expandirse en dos sentidos: en los programas docentes, con acciones que van desde las carreras técnicas, pasando por las profesionales, las licenciaturas y hasta el posgrado, y en la creación de sedes y centros, alcanzando la estructura que muestra la figura 1. 
Figura 1. Estructura de gestión, Universidad Arturo Prat.

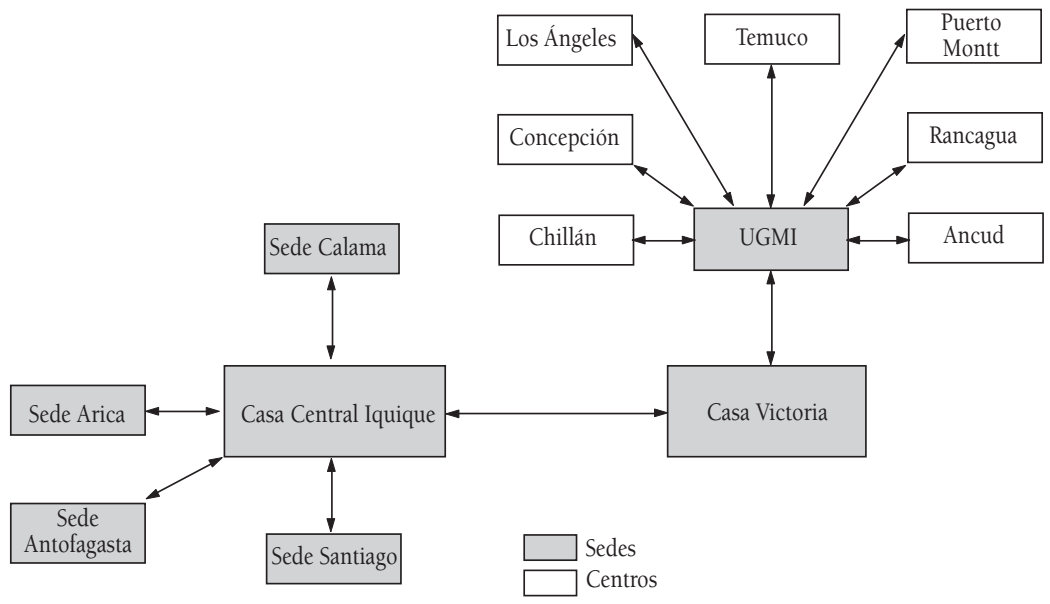

Para respaldar su determinación la universidad analizó una serie de oportunidades, por ejemplo:

- El valor que adquiere el conocimiento en una sociedad globalizada.

- Las exigencias del mercado, en cuanto al requerimiento de nuevas competencias de la población trabajadora del país.

- La gran demanda por educación superior de un segmento de la población que, por la vía tradicional, no ha podido satisfacer sus necesidades formativas.

- Avance de las tecnologías de la información y las comunicaciones.

- Demanda por investigación científica y tecnológica para satisfacer los requerimientos del país y su inserción en la economía mundial.

Enfrentada a este diseño de gestión extendido a todo el país, la universidad ha tenido que desarrollar políticas y mecanismos que aseguran la homogeneidad y calidad docente en todas sus unidades académicas. Estas acciones se expresan a través de la estructura administrativa que gobierna a las sedes y centros, el sistema de administración de carreras, la instalación de un modelo transversal de gestión de la calidad docente y por la 
implementación de recursos de apoyo estandarizados a la docencia para cada centro y sede.

\section{La administración de sedes y centros}

La expansión se encuentra representada a través de dos instancias: las sedes y los centros. La sede, unidad académica en la cual se imparten carreras de diversas disciplinas, posee administración descentralizada para el desarrollo de varias gestiones, académicos de jornada completa y, regularmente, se desarrollan actividades de investigación y extensión académica.

El centro universitario, unidad académica que desarrolla actividad docente en carreras sólo de un área del conocimiento, actúa bajo un sistema de administración académica centralizada para varias acciones de la gestión docente. Pose una estructura administrativa liviana, con escasa autonomía y solamente los académicos de cargos superiores tienen jornada completa. Los centros de la universidad forman una verdadera red que dependen de una unidad académica especializada en metodologías de aprendizaje combinadas (blended learning), llamada Unidad de Gestión de Metodologías Integradas (UGMI).

Como puede desprenderse de la figura 1, la universidad cuenta con dos núcleos de gestión: uno de ellos es la Casa Central, ubicada en la ciudad de Iquique y otra la sede Victoria, ubicada en la IX Región de la Araucanía. En la primera de ellas se monitorea, controla y se toman decisiones sobre las sedes del norte del país (Arica a Santiago). En la sede Victoria se controlan las acciones que se desarrollan en los centros que están ubicados entre Ancud y Rancagua. En esta última sede ocupan un lugar preponderante los centros asociados a UGMI.

Estos dos núcleos tienen la responsabilidad de proveer los recursos financieros y humanos para la operación, inversiones y desarrollo de las unidades académicas bajo su cargo. 
La actividad en las sedes se desarrolla en dos niveles. Las sedes Victoria y Calama para el pregrado tradicional más el pregrado para trabajadores; Arica, Antofagasta y Santiago destinadas a carreras para personas provenientes del mundo laboral. La actividad docente que se lleva a cabo en los centros, en cambio, está diseñada principalmente para este último grupo de estudiantes ${ }^{i}$.

\section{El sistema de administración de las carreras}

\subsection{Creación de carreras}

Todas las carreras se originan en los departamentos de la Casa Central y/o de la sede Victoria. Después de haber alcanzado cierta consolidación, se imparten bajo ciertas consideraciones previas (de docentes y equipamiento) en las otras sedes o centros.

\subsection{La administración de la carrera}

Se ha diseñado un sistema de gestión de carreras que fortalece la capacidad institucional para evaluar y controlar la aplicación igualitaria de las políticas de modernización y estandarización curricular y de administración docente. Existe un jefe de carrera en cada sede donde la carrera es impartida, cuya misión fundamental es la estandarización y homogenización del currículo, su implementación, control y seguimiento, como también de su evaluación y actualización permanente. También, para las carreras que se imparten en más de una sede, hay un coordinador general de carrera, que depende del director de docencia de la Casa Central y es apoyado por un Consejo de Carrera y el Consejo Externo de Carrera. Este último tiene como objetivo disponer de la mirada de empleadores y ex alumnos a fin de ajustar el proceso formativo a las demandas actuales y futuras del mercado laboral (figura 2).

i Cada sede dependiente de la Vicerrectoria Académica de Iquique cuenta con un director de sede, un director de docencia y sus respectivos jefes de carrera. A su vez, los centros dependientes de la vicerrectoría de la sede Victoria dependen directamente del director de la UGMI, que tiene el respaldo de diferentes ejecutivos a cargo de la gestión docente de los centros (jefe de planificación y evaluación docente, supervisores de asignaturas, entre otros); además, en cada centro residen un jefe docente y los respectivos jefes de carrera. 
Figura 2. Sistema de administración de carreras.

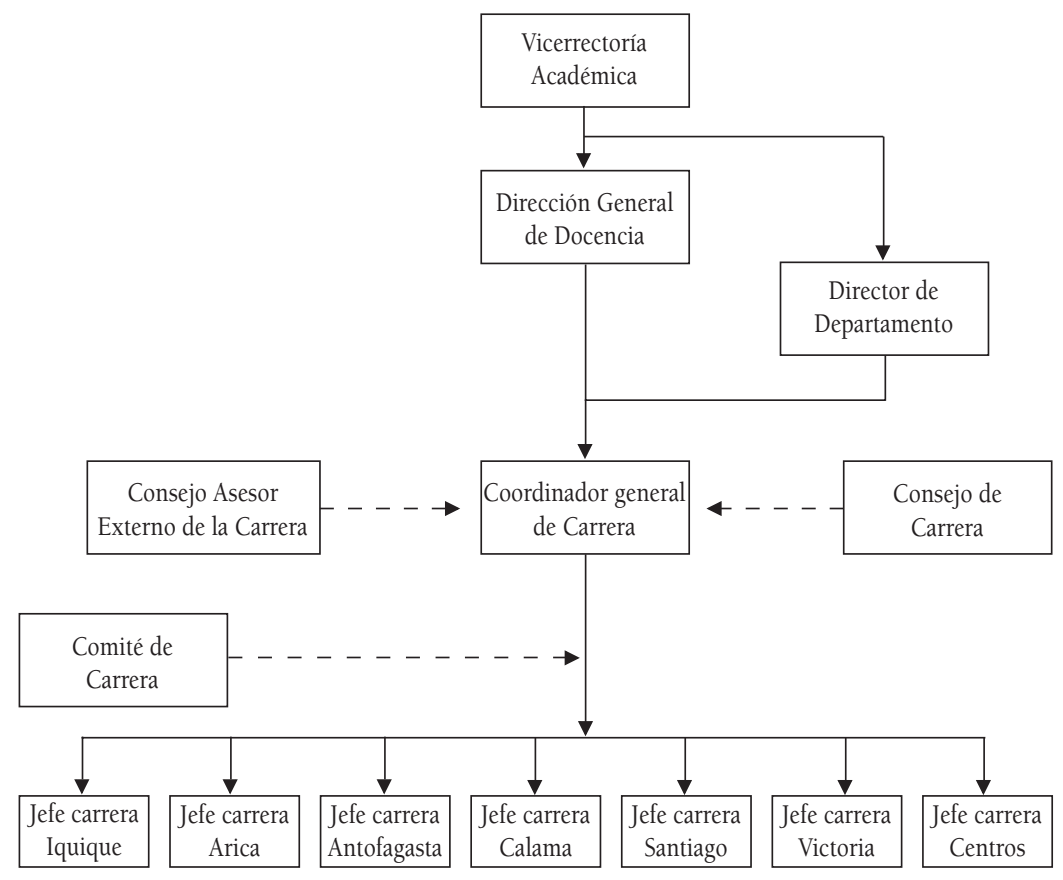

\section{El control de la calidad docente}

Con el fin de contar con un proceso que permita asegurar una buena calidad de la docencia, su mejoramiento continuo y una estandarización y homogeneidad de las carreras y cursos, independiente del lugar en que ellas se entreguen, la Universidad Arturo Prat ha diseñado un modelo de gestión de la calidad. Esta es una herramienta instalada sobre una plataforma informática que cuenta con una serie de sistemas integrados.

Tal como se muestra en la figura 3, este modelo se propone cumplir con seis objetivos. Cada uno de ellos da vida a un proceso que se encadena con otros para formar el modelo de gestión. Los objetivos del modelo comprenden la selección y contrato de los académicos, la clasificación de los académicos por áreas del conocimiento, el control de la homogeneidad de contenidos de las carreras y asignaturas, el 
control de conductas de entrada de los alumnos, el control de conocimientos esperados y la evaluación de la docencia.

Figura 3. Modelo de gestión de la calidad docente.

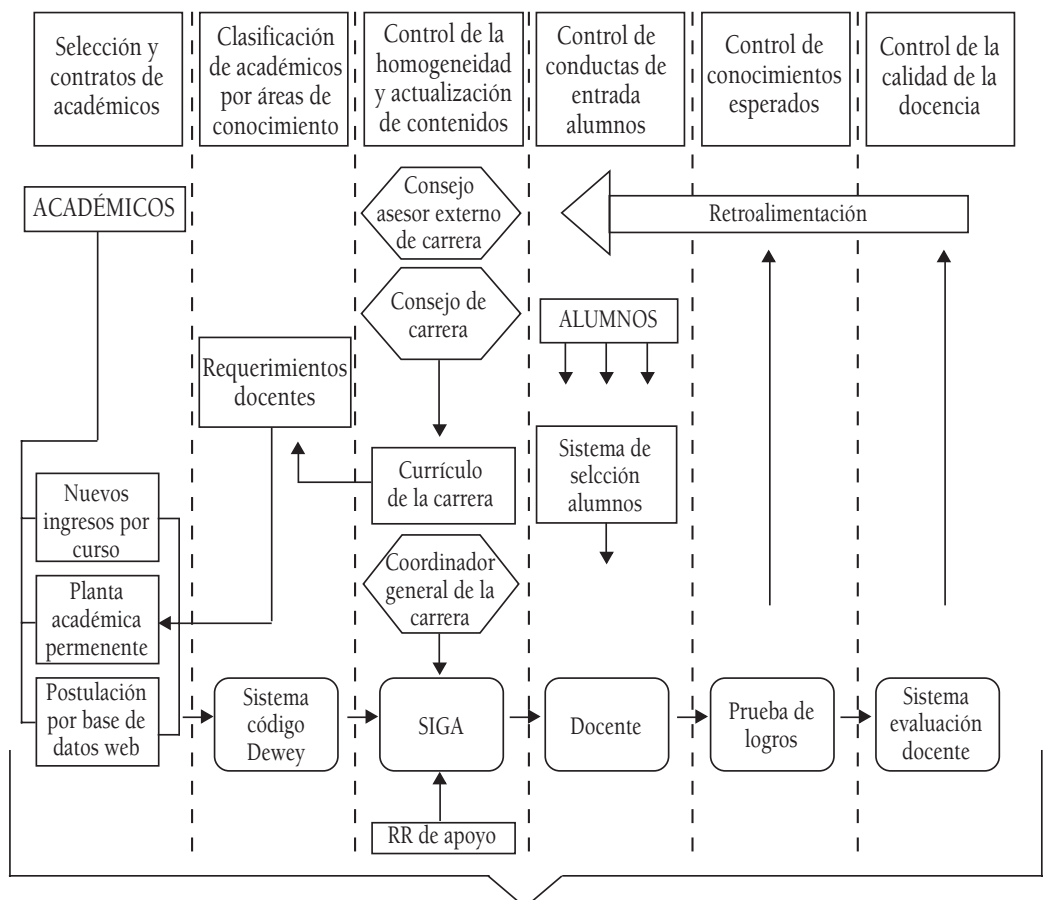

Mejoramiento continuo de la docencia.

\subsection{El proceso de selección y contratos de los académicos}

Luego de la solicitud del jefe de carrera, de acuerdo con los requerimientos del plan de estudio, la selección del académico se puede hacer a través de alguna de estas tres vías: a) revisar las capacidades del personal de jornada completa y media jornada que mantiene la corporación para su elección; b) revisar las bases de datos de concursos anteriores que posee la universidad, ordenadas por área del conocimiento, y c) llamar a concurso público, poniendo en marcha todo el mecanismo que para este efecto tiene la corporación. 
Es interesante señalar también que un número importante de académicos de jornada completa, provenientes de la Casa Central, concurre a las sedes a dictar clases, elemento que coopera con el aseguramiento de la homogeneidad y calidad de la docencia en las sedes.

\subsection{Clasificación de académicos por área del conocimiento}

Para elegir un docente desde nuestras bases de datos (ya sea para jornada completa, media jornada u horas), la universidad asigna la docencia al académico que tenga la mayor pertinencia, esto correlacionando la necesidad docente con la especialización alcanzada por éste.

La universidad ha diseñado, para estos efectos, un sistema de clasificación de los académicos por áreas del conocimiento -utilizando el código Dewey recomendado por UNESCO- donde éstos ingresan sus antecedentes curriculares de docencia, investigación y extensión a una base de datos. Esta herramienta permite presentar hasta tres materias de mayor especialización dentro de su disciplina.

Por otra parte, las asignaturas que imparte la universidad se codifican usando también el sistema Dewey, de manera que se pueden contrastar con los códigos de los académicos, dando así seguridad que se escoge al profesor que tiene la mayor pertinencia para dictar la asignatura.

Además, este sistema entrega al director de docencia y al jefe de carrera toda la información académica del docente, y permite que los académicos de cualquier sede o centro puedan conocer los currículos de sus colegas. Con esto se pretende generar una red de interacción entre académicos de especialidades afines, facilitar el encuentro para el desarrollo de actividades de investigación y extensión, y producir sinergias entre profesores de diferentes sedes y centros.

\subsection{Sistema de Información y Gestión Académica}

Una vez que el profesor ha sido seleccionado por el sistema Dewey, se le hace entrega del Sistema de Información y Gestión académica 
(SIGA) del curso a impartir, donde se encuentra, en forma detallada, la programación del curso: fecha de sesiones docentes, contenidos a tratar por sesión, recursos pedagógicos y rutinas de aprendizaje, y las evaluaciones.

Este sistema permite al profesor generar foros de discusión en relación con determinados temas, responder consultas a través de correos electrónicos e incorporar las notas de los alumnos. Por su parte, los alumnos, con una clave personal, pueden visualizar la planificación de cada uno de sus cursos, conocer las materias por sesión y revisar los materiales. Todo lo anterior orientado a facilitar el aprendizaje y desarrollar una clase presencial constructiva.

Cada asignatura tiene asignado un SIGA, independiente de si se da en diferentes sedes y en diferentes carreras. El responsable de los SIGA de cada carrera es el coordinador general de carrera, quien vela, además, por la homogeneidad docente con independencia del centro o sede en donde esta se imparta.

\subsection{Sistema de selección de alumnos}

Este se desarrolla a través de dos formas: por una parte, está el ingreso tradicional a través del proceso de selección nacional y administrado por el Consejo de Rectores, y por otra, existe el sistema interno de ingresos especiales, orientado principalmente a trabajadores y que exige diferentes competencias de acuerdo con la carrera elegida.

\subsection{Pruebas de logros}

Las pruebas de logros se aplican en diferentes niveles a lo largo de una carrera. Con ellas se pretende contrastar el grado de conocimientos o competencias adquiridas por los alumnos, en diferentes estadios de avance curricular, con los resultados esperados. Permiten conocer el porcentaje de efectividad del proceso de enseñanza-aprendizaje, de manera de tener antecedentes para hacer los ajustes y mejoras necesarios. 


\subsection{Sistema de evaluación docente.}

En la misma plataforma informática, disponible a través de Internet, la universidad mantiene un sistema que considera una evaluación por parte de los alumnos y otra realizada por el jefe de carrera. Existe también un informe de evaluación de la asignatura, mediante el cual el docente, a partir del contexto educativo, evalúa las condiciones en que realiza su docencia en cada una de las asignaturas que imparte y entrega información específica, contrastable con la información recabada en el instrumento que responden los estudiantes. Toda esta información se acopia en una carpeta del docente y permite retroalimentar al sistema.

\section{Los recursos de apoyo a la docencia que cooperan con el aseguramiento de la calidad}

\subsection{Biblioteca}

Como una manera de homogeneizar los elementos que acompañan el proceso docente, se han implementado bibliotecas físicas en todas nuestras sedes y centros, contando estas últimas con un menor número de volúmenes físicos. A fin de asegurar un amplio acceso a bibliografía actualizada se ha incorporado una biblioteca virtual, disponible para los alumnos a través del uso de un sistema de Red Virtual Privada (VPN).

La biblioteca virtual posee, en la actualidad, aproximadamente 24.000 textos suscritos por la universidad, más las bibliotecas libres de Internet con unos 40.000 textos y 15 bases de datos que entregan una gran cantidad de revistas y documentos especializados.

Está disponible, además, el servicio de compra de documentos electrónicos por demanda, que funciona con alta velocidad y satisface necesidades específicas de académicos y estudiantes.

\subsection{Laboratorios}

Todos los centros y sedes mantienen laboratorios de computación abiertos a los alumnos en forma permanente. Algunas sedes poseen 
otros laboratorios especializados como, por ejemplo, física, química y biología, por nombrar algunos en las sedes Iquique y Victoria; los laboratorios de idiomas en Iquique, Santiago y Victoria, los laboratorios de óptica en Santiago.

\subsection{La gestión docente en UGMI}

Como ya se ha mencionado los centros universitarios -orientados a otorgar carreras eminentemente del área de educación-son administrados en forma centralizada desde UGMI. Esta unidad cuenta con una dotación importante de académicos de jornada completa agrupados por especialidades, que tienen por misión construir los textos de clases y los cuadernos de ejercicios y de autoevaluación que por cada asignatura reciben los alumnos. Diseñan también la programación detallada de la docencia y preparan materiales complementarios que se incorporan al SIGA. Es también en UGMI donde se preparan dos de las tres evaluaciones semestrales que tiene cada asignatura, las que se aplican en forma sincrónica en todos los centros.

Es importante consignar que los currículos de las carreras impartidas en los centros son semejantes a las impartidas en las sedes y que, incluso, los SIGA que en ellos se usan son los mismos que siguen los estudiantes del pregrado que ingresan mediante la Prueba de Selección Universitaria (PSU).

\section{5. ¿Hacia dónde vamos?}

Acorde con la política institucional de transformarse en una universidad compleja, se ha diseñado una política que tiende a ir consolidando paulatinamente su presencia en centros y sedes. Es por esto que se hacen gestiones para implementar actividades de investigación y extensión en toda la institución.

En este contexto, la Universidad Arturo Prat ya cuenta con institutos de investigación descentralizados como son los de ciencia y tecnología de Puerto Montt y Concepción. El primero de ellos, con 
casi dos años de vida, opera 15 proyectos financiados por FONDEF, y empieza a acompañar la docencia a través de programas de formación integral para los alumnos. Por otra parte, investigadores del Instituto de Estudios Internacionales de la sede Santiago desarrollan proyectos FONDECYT y preparan nuevas unidades de investigación en Santiago y Los Ángeles, actualmente en su fase de diseño e implementación.

Esas políticas pretenden ir ampliando nuestra participación en este sentido en el corto plazo. Todo esto tendiendo a formar una red universitaria que agrupe diferentes unidades académicas, las que esperamos actúen en forma de anillos intercomunicados, generando alta eficiencia y calidad (figura 4).

Figura 4. Modelo de consolidación de la Universidad Arturo Prat.

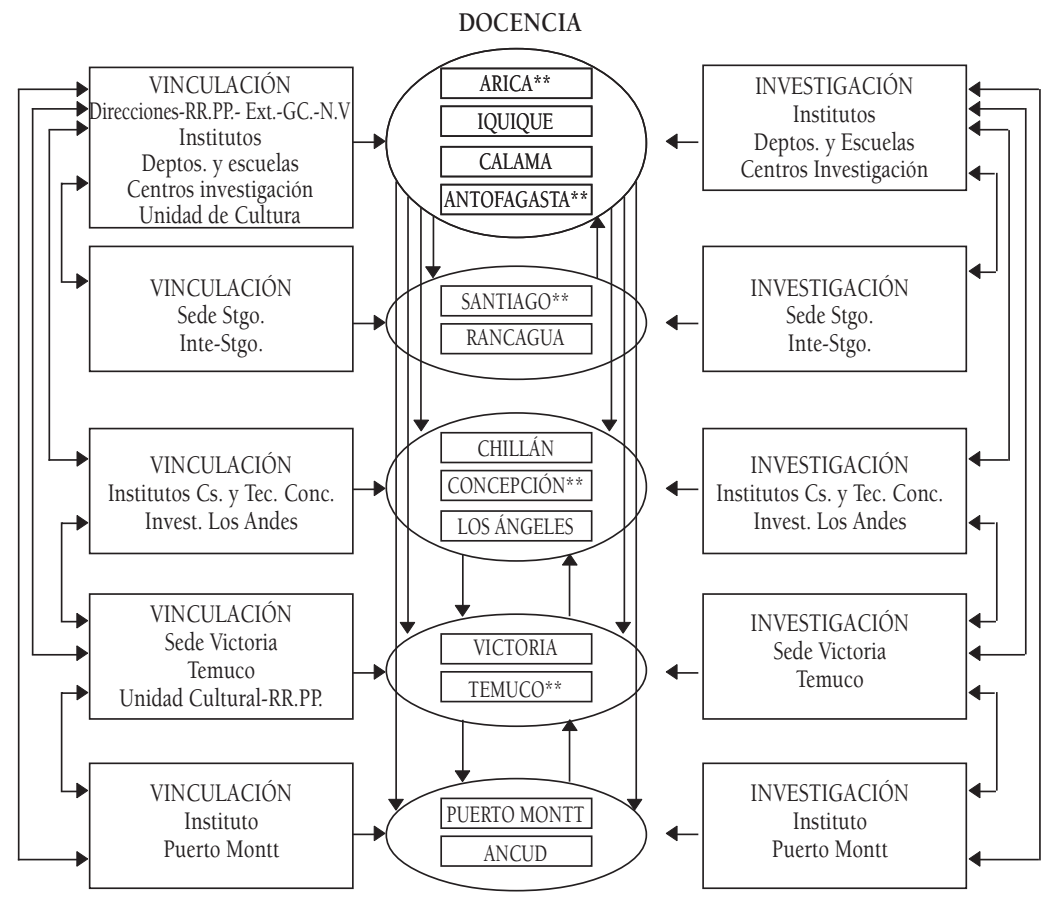

Recibido: 5 de junio de 2006

Aceptado: 15 de junio de 2006 